\title{
Nomenclature Scheme in use by the WGESP and a Current List of Extrasolar Planets
}

\author{
Jill C. Tarter \\ SETI Institute
}

At the IAU General Assembly in 2000, the Working Group on Extrasolar Planets (WGESP) was established as a working group of Division III. Its terms of reference include acting as a focal point for international research on extra solar planets and organizing IAU activities in the field, such as, organizing comparative reviews of techniques used to detect extra-solar planets and establishing criteria for detections of varying degrees of certainty, as well as maintaining lists of objects satisfying these criteria. The committee is chaired by Alan Boss, and the current members are; Paul Butler, William Hubbard, Philip Ianna, Martin Kürster, Jack Lissauer, Michel Mayor, Karen Meech, Francois Mignard, Alan Penny, Andreas Quirrenbach, Jill Tarter, and Alfred Vidal-Madjar.

The activities of this working group are reported on the web site

http://www. ciw. edu/IAU/div3/wgesp/planets.shtml.

In particular, the members of the working group have established a working definition for a body called a "planet", and have created a list of objects that satisfy that definition. This definition (as last modified on Feb. 28, 2003) can be found at

http://www . ciw.edu/boss/IAU/div3/wgesp/definition. shtml,

and can be expected to be modified as we improve the census of low-mass stellar companions.

The most salient points about what does and does not constitute a planet are as follows. A planet must:

- have a true mass below the deuterium burning limit $\left(\sim 13 M_{J}\right.$ for solar metallicity),

- orbit a star or stellar remnant, and

- have a true mass above whatever we end up adopting for our own solar system.

Note that this definition specifically excludes "free-floaters." Until there is an unambiguous determination that any such body was born in orbit around a star or stellar remnant, then they will be classified on the basis of their estimated 
mass. Objects with substellar masses exceeding $13 M_{J}$ will be referred to as Brown Dwarfs, while those with masses less than $13 M_{J}$ will be called subBrown Dwarfs.

The current list of objects satisfying this working definition can be found at:

http://www.ciw.edu/boss/IAU/div3/wgesp/planets.shtml.

At present, the list contains 99 entries, all of which have been detected by radial velocity techniques. Since true masses are not available from this technique alone, the working list consists of reported planets with $M \sin i<10 M_{J}$. The format for the current table uses the Hipparcos, and HD designations and common name of the star as the primary indicator. The date of receipt of the discovery paper by the refereed journal that eventually published the discovery is also cited, and is a requirement for inclusion in the working list. A second table of pulsar planets (explicitly permitted under the working definition) can be found on the same web page.

Although it is tempting to invent or adopt some "popular" names for the individual extra-solar planets (particularly in interactions with the media), the WGESP has specifically resisted this temptation. The WGESP further agrees that it will uses whatever new classification scheme is adopted by the IAU for double star systems, but that the working group does have a natural preference for a nomenclature that recognizes the difference between a planet and a star.

\section{Discussion}

POURBAIX: Could you add the ADS bibcode to your table?

TARTER: I see no reason why the table should not have links to the ADS bibcode. Good suggestion.

DICKEL: (Suggestion) 1. Initially the nature of the component of a multiple star system may not be known - i.e. whether it is a planet or star in the WMC catalog. 2. When the component is discovered to be a "planet", it goes into the official extra-solar planet list with an Exoplanet type "acronym" or designation. TARTER: Good suggestion. I will talk with you about the approved acronym we should adopt.

ZINNECKER: Do you think that the definition of a planet that the IAU Working Group has proposed will survive? I sense some disagreement in the community.

TARTER: The definition of planet adopted by the WGESP22 is a "working" definition and will change over time if the community changes its mind. There was significant debate over each point prior to reaching the current consensus. We will always keep the working list of planets consistent with the working definition of planets.

\footnotetext{
${ }^{22}$ Working Group for Extra-Solar Planets
} 
ZINNECKER: Many of the free-floating objects will been born as planets, for example, in binary systems, but ejected. What should we call them?

TARTER: Until we find some unambiguous marks that identified the birth place of a "free floater" as having been in orbit around a star or stellar remnant, we have chosen not to list them, and on basis of mass, call them brown dwarfs or sub-brown dwarfs.

TOKOVININ: A preference to a designation scheme that makes a difference between stellar companions or planets is not practical; some "planets" with $M \sin i<10 M_{J}$ will be stars, some with $M \sin i>10 M_{J}$ will have $M<13 M_{J}$. TARTER: True. What I meant to say was that it would be desirable to have the designation scheme reflect the fact that with the knowledge in hand, the object was currently listed on the WGESP working list of planets. Over time, the designations might have to change to accommodate new information. I understand that gets into the middle of the "static" vs. "dynamic" argument. Rather than take sides, I merely stated a small preference. 